
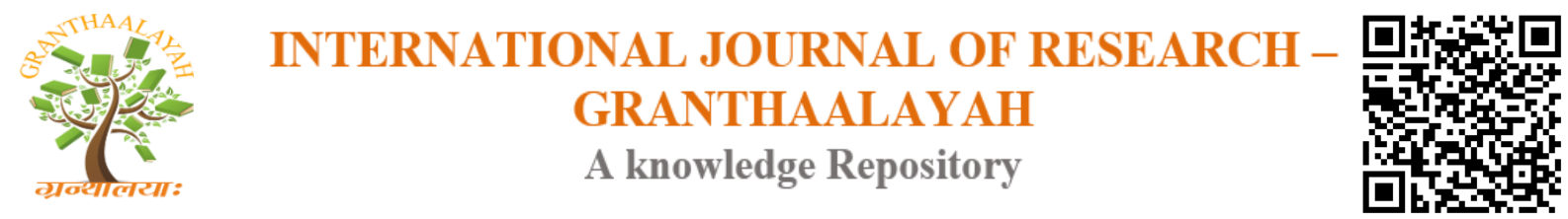

Science

\title{
THE ROLE OF SOFTWARE IN A CASHLESS ECONOMY (CASE STUDY NIGERIA)
}

\author{
Sese Tuperekiye Ebimobowei ${ }^{1}$, Zoukemefa Enebraye Peter ${ }^{2}$, Yerikema Pual (Jnr) ${ }^{3}$ \\ ${ }^{1,3}$ Department of Computer Science, Bayelsa State College of Arts and Science P.M.B 168 \\ Elebele Yenagoa \\ ${ }^{2}$ Department of Computer Science, Isaac Jasper Boro College of Education Sagbama, Bayelsa \\ State
}

\begin{abstract}
In the world today most country's economy is gradually going cashless and Nigeria is no exception. In other for this to be achieved the use of computers and computerized networks and its software comes greatly into play. In an attempt to make the country's economy cashless driven, we will discover that software plays a prominent role and can only be unveiled when we take a close study of how some of these payment mechanisms functions. Such mechanisms include POS TERMINALS, smart mobile phones and computers (laptop/desk top). There are basically two categories of software are installed on each of these devices; they include an operating system and application software. Also this paper went the extra mile of explaining what cashless economy is, the aim of establishing the policy in Nigeria and the benefits.
\end{abstract}

Keywords: Cashless; Automated Teller Machine (ATM); Point-of-Sale (POS); Central Bank of Nigeria (CBN); Online Payment System (OPS); Network; Mobile Smart Phones.

Cite This Article: Sese Tuperekiye Ebimobowei, Zoukemefa Enebraye Peter, and Yerikema Pual (Jnr). (2018). "THE ROLE OF SOFTWARE IN A CASHLESS ECONOMY (CASE STUDY NIGERIA)." International Journal of Research - Granthaalayah, 6(1), 177-186. https:// doi.org/10.29121/granthaalayah.v6.i1.2018.1607.

\section{Introduction}

As the need for a cashless economy rises, people are increasingly seeing the need to use computer networks to access and pay for goods and services with e-money (E-money or digital cash is merely an electronic representation of funds). The primary function of e-cash or e-money is to facilitate transaction on the network. For e-money to become fully functional, some infrastructures need to be put in place. These infrastructures could be Smart phones, POS terminals etc. therefore in attempt to unveil the role of software in a cashless economy, I will analyze the functionalities of these infrastructures to show the connection they have with software. 


\section{What Is A Cashless Economy}

A cashless economy is a system where all means of payments are carried out without the use of physical cash. Payment will range from a list of options such as cheques, wire transfers, debit and credit cards, online transactions and mobile banking.

In Nigeria, cashless economy has been introduced with the pilot phase that started in Lagos since January 2012. The new policy on cash-based transactions (withdrawals \& deposits) in banks, aims at reducing (NOT ELIMINATING) the amount of physical cash (coins and notes) circulating in the economy, and encouraging more electronic-based transactions (payments for goods, services, transfers, etc.).

Initially, the banking sector regulator fixed the daily cumulative cash withdrawal/deposit limit for individual accounts to be N150, 000.00 per day and corporate accounts to be N1 million. Apparently responding to public outcry over the daily ceiling on cash withdrawals and deposits, the Central Bank of Nigeria $(\mathrm{CBN})$ has raised the bar from the previously announced amount to N500,000 for individual account and N3 million for corporate account.

This goes a long way to show that physical cash will still be handled only that the dominance will be reduced.

\section{The new cash policy was introduced for a number of key reasons, including:}

1) To drive development and modernization of our payment system in line with Nigeria's vision 2020 goal of being amongst the top 20 economies by the year 2020. An efficient and modern payment system is positively correlated with economic development, and is a key enabler for economic growth.

2) To reduce the cost of banking services (including cost of credit) and drive financial inclusion by providing more efficient transaction options and greater reach.

3) To improve the effectiveness of monetary policy in managing inflation and driving economic growth.

In addition, the cash policy aims to curb some of the negative consequences associated with the high usage of physical cash in the economy, including:

- High cost of cash: There is a high cost of cash along the value chain - from the CBN \& the banks, to corporations and traders; everyone bears the high costs associated with volume cash handling.

- High risk of using cash: Cash encourages robberies and other cash-related crimes. It also can lead to financial loss in the case of fire and flooding incidents.

- High subsidy: $\mathrm{CBN}$ analysis showed that only 10percent of daily banking transactions are above $150 \mathrm{k}$, but the 10percent account for majority of the high value transactions. This suggests that the entire banking population subsidizes the costs that the tiny minority 10percent incurs in terms of high cash usage.

- Informal Economy: High cash usage results in a lot of money outside the formal economy, thus limiting the effectiveness of monetary policy in managing inflation and encouraging economic growth. 
- Inefficiency \& Corruption: High cash usage enables corruption, leakages and money laundering, amongst other cash-related fraudulent activities.

A variety of benefits are expected to be derived by various stakeholders from an increased utilization of e-payment systems. These include:

- For Consumers: Increased convenience, more service options, reduced risk of cashrelated crimes; cheaper access to (out-of-branch) banking services and access to credit.

- For Corporations: Faster access to capital, reduced revenue leakage, and reduced cash handling costs.

- For Government: Increased tax collections; greater financial inclusion; increased economic development.

\subsection{Is It Achievable?}

I would answer yes to that.

First we should note one of the hindrances to this development is people's mindset. With every new endeavor in life, one will always have resistance; there will be pockets of resistance or pushback. The reason is that it is the nature of humans to remain in their comfort zone, we like to operate where we are comfortable. That is the first hurdle we are going to cross.

In order to do this, the CBN has been running a set of targeted stakeholder engagement sessions as a first stage of its planned communication campaign. These have targeted key groups that will be most impacted by the cash policy, with the objective of creating awareness and providing an opportunity for them to raise issues and concerns. The stakeholders so far have included markets, associations, professional bodies, etc. These stakeholder sessions were concluded in November 2011. CBN is currently implementing the phase 2 of the communication strategy, which is the Mass communication campaign, leveraging the inputs derived during the stakeholder sessions.

And once people see that the system works and the hassles of having to take your cash to the bank everyday is eliminated, I think the issue of push backs or resistance will be addressed.

Then there is the issue of infrastructure readiness, power, lack of POS terminals, insufficient and inadequate ATM machines etc. we all know that the man that continue to observe the wind will not sow. In the case of power, must we have power 24/7 before we roll out the cashless society? I say no! Today we are not all dead because there is no power, we should run with the alternatives that we have and simultaneously we keep pushing for improvement on the power front so that as the power keeps improving the system will keep getting better.

\section{Role of software in achieving cashless economy}

We cannot say there is particular software which provides the leverage for a cashless economy so that we should begin to analyze it and look for whom and where it is developed. Since the aim of a cashless economy is to reduce the dominance of raw cash in circulation, we are therefore going to examine some payment mechanisms in order to determine the role of software in a cashless economy; we need a proper analysis of the devices to be used. Below are the lists of devices to be used. 
1. POS terminal

2. ATM

3. PDA (Mobile smart phones etc.)

\subsection{POS System}

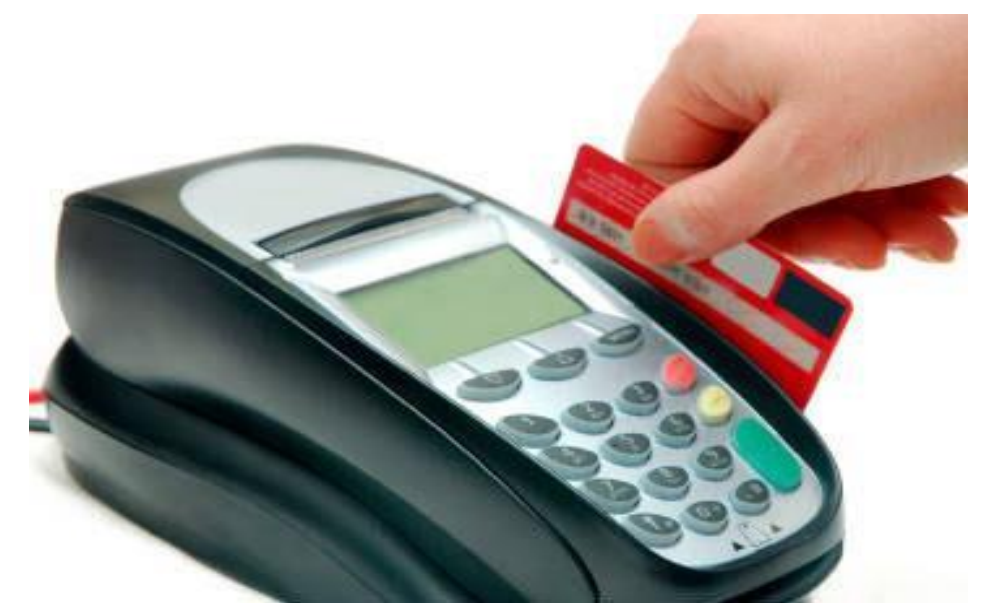

Point-of-sale (POS) systems are methods and programs that are used to assist merchants in the sale of retail goods and services. The term comes from the description of the very moment that a customer or consumer makes the exchange of money with the merchant for the goods or services.

A point-of-sale (POS) terminal is a computerized replacement for a cash register. Much more complex than the cash registers of even just a few years ago, the POS system can include the ability to record and track customer orders, process credit and debit cards, connect to other systems in a network, and manage inventory. Generally, a POS terminal has as its core a personal computer, which is provided with application-specific programs and I/O devices for the particular environment in which it will serve.

A POS system for a restaurant, for example, is likely to have all menu items stored in a database that can be queried for information in a number of ways. POS terminals are used in most industries that have a point of sale such as a service desk, including restaurants, lodging, entertainment, and museums.

Running a restaurant is no easy task. Everything from the ordering and preparing of food to Procuring reservations needs immense attention to detail.

Luckily, through the advent of restaurant POS software, some of these tasks have become a little easier. Restaurant POS software keeps track of everything from reservations to inventory to sales figures. Here are some key terms commonly found when interfacing with restaurant POS software. 


\section{Central Office Reporting}

Central office reporting refers to a function of restaurant POS software that reports inventories and financial statistics to the home office. This is most commonly used in chain restaurants so that the franchise headquarters and franchise locations can stay in sync. Try: Visit the website for Point of Success. This is a manufacturer of restaurant POS software and has a comprehensive page explaining central office reporting.

\section{Table Diagram}

A table diagram is found in virtually all restaurant POS software systems for any cafe style or fine dining restaurant. The diagram exhibits all of the tables in the restaurant, as well as the number of patrons at each of those tables. Many table diagrams interact with a touch screen. Try: Visit PC America for further information about the functionality of table diagrams found within restaurant POS software.

\section{Time and Attendance}

The time and attendance feature refers to an application within the software that calculates when employees are working and how much they are selling. Employees log into the software using a code and the time and attendance feature keeps track of their sales. More advanced applications also track tips and prepare tax forms.

Try: Maitre'time and attendance feature keeps track of their sales. More advanced applications also traD POS makes restaurant solution software and has a detailed page describing time and attendance.

\section{Inventory Control}

Inventory control refers to an application that tracks shipments. Inventory control is imperative to the success of a restaurant so that they can keep their food margins monitored. Try: Visit VIP Solutions for further information on inventory control.

\section{Integrated Ordering System}

An integrated ordering system is an application within restaurant POS software that allows customers to place orders through Internet ordering, phone ordering, kiosk ordering or ordering at any other location. The order is sent to both the cashier and the kitchen so that the food may be prepared.

Try: Speedline Solutions has further information about integrated ordering systems.

\section{Sales Report}

A sales report refers to a calculation over a period of time that determines the gross sales of the restaurant. All restaurant POS software programs have some form of this application. The more in-depth applications track trends, popular items and customer favorites. Try: Visit the website for Sixth Sense POS for further information about the use of sales reporting within restaurant POS software.

Am not saying a POS is only applicable in restaurant, there are other area were POS can be applied like Hotel business POS software which allows for transfer of meal charges from dining 
room to guest room with a button or two, also in Hair and beauty industry In order to run a salon efficiently it is essential to keep all appointments, client, employee roster and the checkout in a system where you can create performance reports for e.t.c.

\subsection{ATM (Automated Teller Machine)}

The ATM is another powerful tool that will be relevant in a cashless economy. ATM provides money withdraw, deposit, money transfer, account balance, management services e.t.c. It is divided into two parts: the hardware \& software.

The hardware is further divided into nine parts

1. CPU (to control the user interface and transaction devices).

2. Magnetic and/or Chip card reader (to identify the customer).

3. PIN Pad (similar in layout to a Touch tone or Calculator keypad), often manufactured as part of a secure enclosure.

4. Secure cryptoprocessor, generally within a secure enclosure.

5. Display (used by the customer for performing the transaction).

6. Function key buttons (usually close to the display) or a Touchscreen (used to select the various aspects of the transaction).

7. Record Printer (to provide the customer with a record of their transaction).

8. Vault (to store the parts of the machinery requiring restricted access).

9. Housing (for aesthetics and to attach signage to).

But let's focus our attention on the software where aspect of the ATM because that is what this research is all about. With the migration to commodity Personal Computer hardware, standard commercial "off-the-shelf" operating systems, and programming environments can be used inside of ATMs. Typical platforms previously used in ATM development include $\underline{\text { RMX }}$ or $\underline{\text { OS/2 }}$.

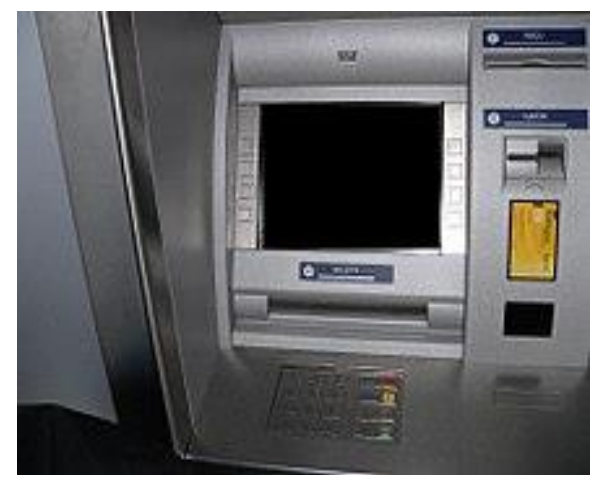

A Wincor Nixdorf ATM running Windows 2000

Today the vast majority of ATMs worldwide use Microsoft Windows OS, primarily Windows XP Professional or Windows XP Embedded. A small number of deployments may still be running older versions of Windows OS such as Windows NT, Windows CE, or Windows 2000. 
There is a computer industry security view that general public desktop operating systems have greater risks as operating systems for cash dispensing machines than other types of operating systems like (secure) real-time operating systems (RTOS). RISKS Digest has many articles about cash machine operating system vulnerabilities. ${ }^{[34]}$

$\underline{\text { Linux }}$ is also finding some reception in the ATM marketplace. An example of this is Banrisul, the largest bank in the south of Brazil, which has replaced the MS-DOS operating systems in its ATMs with Linux. Banco do Brasil is also migrating ATMs to Linux.

Common application layer transaction protocols, such as Diebold 91x (911 or 912) and NCR NDC or NDC+ provide emulation of older generations of hardware on newer platforms with incremental extensions made over time to address new capabilities, although companies like NCR continuously improve these protocols issuing newer versions (e.g. NCR's AANDC v3.x.y, where $\mathrm{x} . \mathrm{y}$ are subversions). Most major ATM manufacturers provide software packages that implement these protocols. Newer protocols such as IFX have yet to find wide acceptance by transaction processors. [35]

With the move to a more standardized software base, financial institutions have been increasingly interested in the ability to pick and choose the application programs that drive their equipment. WOSA/XFS, now known as CEN XFS (or simply XFS), provides a common API for accessing and manipulating the various devices of an ATM. J/XFS is a Java implementation of the CEN XFS API.

While the perceived benefit of XFS is similar to the Java's "Write once, run anywhere" mantra, often different ATM hardware vendors have different interpretations of the XFS standard. The result of these differences in interpretation means that ATM applications typically use a middleware to even out the differences between various platforms.

With the onset of Windows operating systems and XFS on ATM's, the software applications have the ability to become more intelligent. This has created a new breed of ATM applications commonly referred to as programmable applications. These types of applications allows for an entirely new host of applications in which the ATM terminal can do more than only communicate with the ATM switch. It is now empowered to connected to other content servers and video banking systems.

Notable ATM software that operates on XFS platforms include Triton PRISM, Diebold Agilis EmPower, NCR APTRA Edge, Absolute Systems AbsoluteINTERACT, KAL Kalignite, Phoenix Interactive VISTAatm, and Wincor Nixdorf ProTopas.

NOTE: RMX or OS/2, Windows 2000, Windows XP Professional or Windows XP Embedded, Windows NT, Windows CE, Linux are all operating system while XFS, Triton PRISM, Diebold Agilis EmPower, NCR APTRA Edge, Absolute Systems AbsoluteINTERACT, KAL Kalignite, Phoenix Interactive VISTAatm, and Wincor Nixdorf ProTopas are the application programs or software needed to drive the entire ATM hardware. 


\section{Online Payment System}

Electronic payment is a financial exchange that takes place online between buyers and sellers. All a person would need is an Internet enabled environment, your personal computer, for an organization a server computer and that is all you require.

Starting from the notebook or laptop, it is very pertinent that we realize that there must an operating system which is a software that must be installed to coordinate the activities of the computer to be used as well as creating an enabled environment were software relevant to online transactions can run on.

A case study is blueberry software which is a sister of blueberry consultant, selling the popular BB flashback screen recorder worldwide. Over the past few years, blueberry has developed the BB software website into a sophisticated online payment sytem.

System support includes:

- Customer registration.

- Customer product selection, including quantity discount and site licenses.

- Discount Coupons at point of sale.

- Order progress through email communication to customers.

- License key generation and validation.

- Electronic invoice/receipt delivery in pdf plus credit note support.

- Multi-currency support.

- Full VAT handling at point of purchase.

Effectively, this is a complete software system for running software sales business.

Note - this system is a custom development for blueberry software, but it could be adapted for use by another company relatively quickly.

This definitely is resident in a remote computer or host server somewhere so that through the help of the internet, it is possible for a customer to access from anywhere in the world. The idea is that in order to pay for software, one does not need to carry physical cash. Through use of credit cards, visa cards etc. it becomes very possible to do business without need to carry cash. Majority of existing companies and businesses have websites that always for online cash transactions to be carried out.

\subsection{Smart Mobile Phones}

Many service providers of cellular networks are offering the convenience of paying bills with mobile smart phones. In such a mobile electronic payment framework, the participants of mobile payment involve the customer, merchant, payment gateway, issuer bank, and acquirer bank. A good example in Nigeria is the MOBILE MONEY and Mobile Banking solution supplied by Guaranty Trust Bank (GTB) in partnership with Fortis Micro Finance Bank and powered by MTN. 
The customer is equipped with a secure smart phone. Such a secure smart phone serves as ewallet, and the mobile payment application is loaded into the smart phone as a trusted application: before running the application, the Operating System (examples include Windows ce, SymbianOS) verifies the integrity and trustworthiness of the mobile payment application with the aid of TPM (Trusted Platform Module); only when the verification succeeds, may the mobile payment application be invoked. Security of the mobile payment application is further enforced by the OS, which implements the reference monitor concept.

The secure smart phone may choose one of the following payment channels:

- TCP/IP channel over the Internet;

- A WAP channel over cellular network, such as GSM network;

- Short range radio channel (such as Bluetooth technology), connected to POS terminal or vending machine.

So, be it an ATM or a POS terminal, mobile smart phones etc. the general e-payment scheme is represent by the diagram below. All e-payment mechanism must have software or applications that will allow for transaction to and fro the host server.

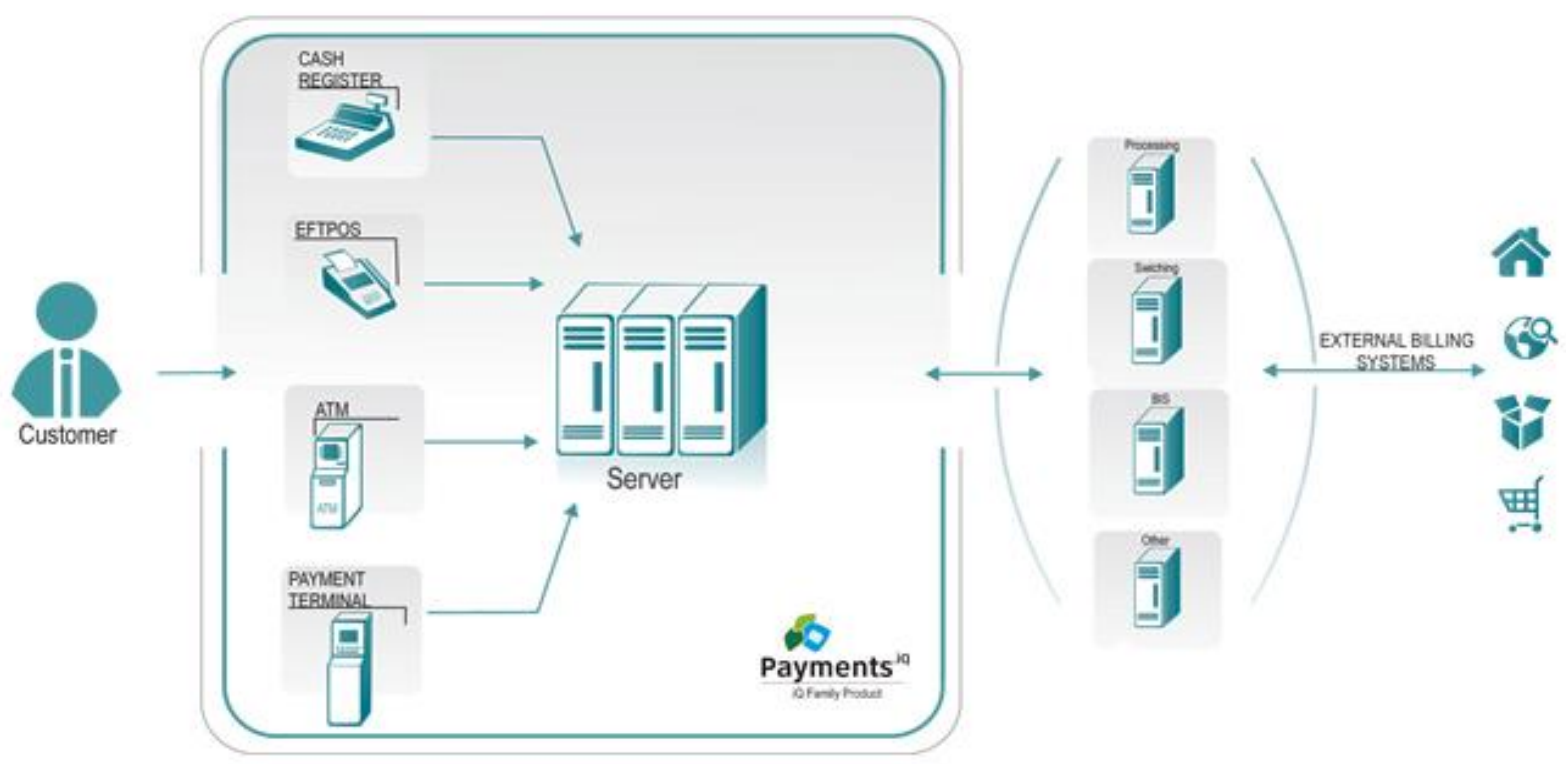

\section{General E-Payment Scheme}

\section{References}

[1] Apacs (2006) 'The Way We Pay 2006', UK Cash \& Cash Machines Highlights.

[2] ATM: ad/i-design.

[3] The IFX Standard (2006) 'GlobalAdoption is Only a Matter of Time',CELENT LLC, May TCG: TCG Specification Architecture Overview, Revision 2.1, 28 April 2004.

[4] Vesna Hassler: Security Fundamentals for E-commerce, Artech House, 2001

\section{Web References}

[1] http://www.cenbank.org/ 
[2] http://www.cenbank.org/cashless/POS_GUIDELINES_August2011_FINAL_FINAL\%20(2).pdf

[3] http://www.cenbank.org/cashless/Cashless\%20Lagos\%20Presentation_November.pdf

[4] http://docs.oracle.com/cd/B31446_01/Point-of-Sale/pdf/72/pos-720-devg.pdf

[5] http://www.ehow.com/about_5686098_different-types-pos-system_.html

[6] http://en.wikipedia.org/w/index.php?title=Salon_software\&action=edit\&redlink=1

[7] http://www.dgca.gov.al/ESARR-6.pdf

[8] http://en.wikipedia.org/wiki/Automated_teller_machine

*Corresponding author.

E-mail address: tupsese@ yahoo.com/ pjyeri@yahoo.com 